\title{
The Importance of Oral Health in the Older Patient
}

\author{
Kenneth Shay, DDS, $M S^{* t \neq}$ and Jonathan A. Ship, DMD
}

Oral health is important to general health because stomatologic disease affects more than the mouth. Increasing preservation of teeth among present and future cohorts of older people has increased their risk for serious disease from oral pathogens. The intent of this paper is twofold: first, to alert non-dental health personnel to the significance of oral health and oral disease in the older adult; and second, to recruit the assistance of non-dental professionals in helping patients to achieve and maintain an optimal oral condition. Normative aging processes alone have little effect on the oral cavity, but common disease processes affecting oral health include tooth loss, dental caries, periodontal diseases, and oral mucosal diseases (including candidiasis and squamous cell carcinoma). Systemic diseases and their treatments frequently affect salivary, oral motor, and oral sensory functions. As a result of bacteremia or aspiration of oral contents, organisms of oral origin can be responsible for serious nonstomatological infections. Clinicians caring for older people need to recognize the importance of stomatological health, include an oral component in the multidisciplinary geriatric assessment, support the education of patients on aspects of dental health, and advocate the expansion of personal and public oral health benefits for older adults. J Am Geriatr Soc 43:1414-1422, 1995.
From the "Dental Service, Ann Arbor VA Medical Center; tGeriatric Research, Education, and Clinical Center, Ann Arbor VA Medical Center; and $\ddagger$ Department of Oral Medicine, Pathology, and Surgery, University of Michigan School of Dentistry, Ann Arbor, Michigan.

Address correspondence to Kenneth Shay, DDS, MS, Chief, Dental Service (160), VA Medical Center, Ann Arbor, MI 48105.
Three essential physiological tasks are undertaken by the oral cavity: initiation of alimentation, production of speech, and host protection. Intake of nutrients is begun by chewing and mixing the food with saliva and then manipulating that combination into a bolus, transporting it to the pharynx, and initiating the swallowing process. Speech depends on delicate and precise positioning of teeth, lips, cheeks, and tongue; many nonverbal components of interpersonal communication revolve around the mouth and lower face as well. Finally, the airway and alimentary canal are protected by salivary secretions, as well as by proper functioning of the swallowing mechanism. These activities are important at all ages, but particularly in older people, who may be predisposed to malnutrition, whose social contacts take on growing importance, and whose susceptibility to infection is of particular concern. ${ }^{1}$

Unfortunately, the health of the oral cavity and its ability to fulfill its functions is commonly impaired in older adults. ${ }^{2}$ Age alone does not seem to play a strong role in the impairments. ${ }^{3}$ Rather, loss of one or more teeth as a result of disease can predispose to further tooth loss, ${ }^{4}$ destruction of alveolar bone, ${ }^{5}$ dependence on and compromised function of prosthetic replacements, ${ }^{6}$ and mucosal disease. ${ }^{7}$ The principal processes responsible for tooth loss-dental caries and periodontal diseases-also lead to sensitivity, pain, and impaired chewing and speaking ability. Lesions of the soft tissues of the mouth can interfere with mastication and oral motor function and can affect nutritional status. ${ }^{8}$ In extreme cases, such as squamous cell carcinoma, extreme morbidity and even mortality follow. ${ }^{9}$ Many of the key protective mechanisms of the oral cavity are compromised when saliva is altered or diminished by medications, irradiation, or disease. ${ }^{10}$ Furthermore, oral motor ${ }^{11}$ and oral sensory dysfunctions are associated with salivary gland dysfunction as well as with medical conditions and therapies prevalent in old age. ${ }^{12}$

The effects of stomatological diseases are not limited to the oral cavity and its functions. Oral diseases give rise to pathogens, which can be blood borne ${ }^{13}$ or aspirated into the lungs, ${ }^{14}$ bringing about severe, even life-threatening consequences. Today's older adult is much more likely to have natural teeth compared with previous aged cohorts ${ }^{15}$ (Figure 1). Thus, older persons are currently at higher risk of developing serious dentally derived systemic disease than were earlier cohorts of elders.

Preserving oral health and avoiding stomatologic diseases should, therefore, be of interest to all health providers who care for older persons. The intent of this paper is, first, to alert non-dental health personnel to the significance of oral health and oral disease in the older adult. Second, it seeks to 


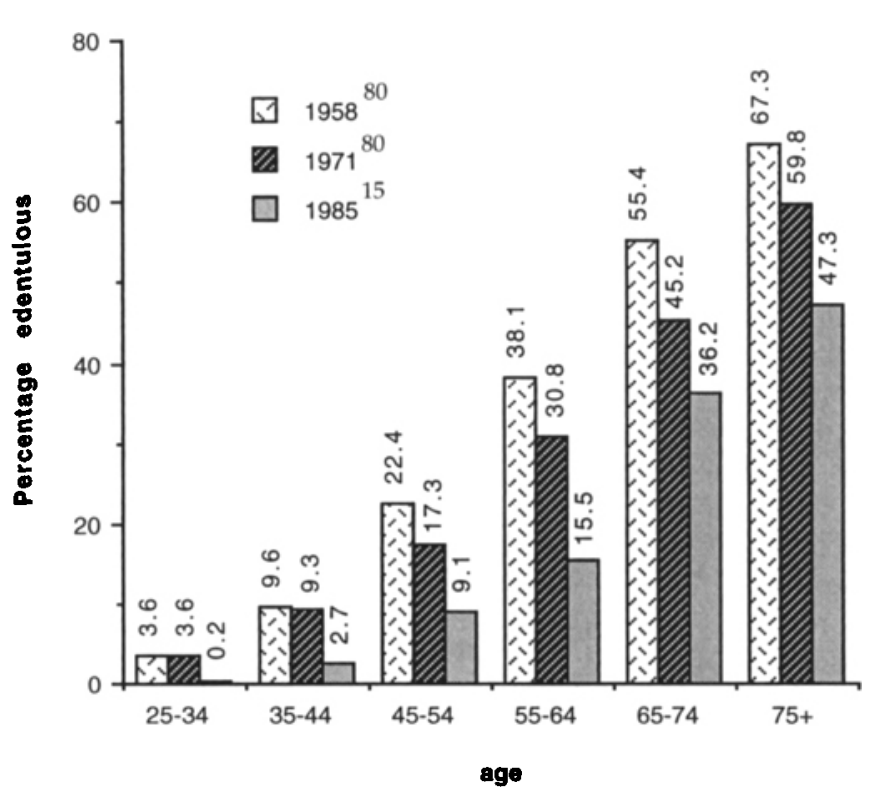

Figure 1. Edentulousness in the US adult population by age group, 1958, 1971, and 1985 .

recruit the assistance of non-dental professionals in helping patients to achieve and maintain an optimal oral condition.

\section{ORAL HEALTH AND ORAL DISEASE}

Many specialized tissues have evolved in the mouth to fulfill functions related to nutrition, communication, and host protection (Table 1). Whereas some of these tissues undergo age-related changes, others can remain remarkably intact in healthy, older individuals. ${ }^{3}$ Impairment in any of these tissues can, of course, bring about oral problems; to describe the contributions of these problems to systemic diseases is one of the goals of this paper.

\section{Tooth Loss}

The loss of teeth, an irreversible and cumulative process, has long been associated with aging. Yet the major portion of tooth loss is attributable to two preventable diseases: dental caries and periodontal diseases. ${ }^{16}$ The 1985 National Institute of Dental Research (NIDR) National Survey of Oral Health in US Adults reported that more than $41 \%$ of Americans older than age 65 were edentulous (total absence of teeth) and that those older than 65 who retained one or more teeth had an average of 11 missing teeth. ${ }^{15}$ However, the prevalence of edentulous adults has dramatically decreased since the first National Health Survey in 1957-58 ${ }^{17}$ (Figure 1), and projections indicate that this trend will continue as older people retain more teeth than previous cohorts. This retention of teeth into advanced age is the result of greater retention earlier in life, advances in dental treatment and disease prevention, improved self-care behaviors, increased availability of dental care, and growing awareness of dental needs. $^{18}$

Missing teeth can have profound effects on both oral and systemic health. Uncompensated tooth loss results in tipping and supereruption of other teeth, leading to food impaction, predisposition to dental and periodontal diseases (see below), impaired chewing, and further tooth loss. ${ }^{4}$ Partial or complete edentulism disturbs the preparation of food for deglutition $^{19}$; food selection can be altered, leading to a softer, carbohydrate-rich diet lacking in fiber and protein. ${ }^{20,21} \mathrm{~A}$ recent population-based sample of older people found that edentulousness was correlated with lower nutrient intakes and multiple dietary inadequacies, and edentulous individuals were more likely to have an atherogenic diet. ${ }^{22}$ In the absence of teeth (with or without dentures), the discrimination of food particles using textural and tactile perceptions may become impaired, ${ }^{2.3}$ and this could alter swallowing and predispose an individual to aspiration episodes. ${ }^{24}$

The quality of a person's life can be dramatically affected by edentulism, even when replacement prostheses are worn. Partial and complete dentures are imperfect replacements for teeth and periodontal ligaments; chewing, swallowing, speaking, facial aesthetics, and social interactions are all potentially affected. ${ }^{6}$ This is particularly true for individuals who have been without teeth for a prolonged time because loss of alveolar bone in the edentulous jaws continues even decades after the removal of natural teeth. ${ }^{5}$ The resulting advanced alveolar ridge resorption is associated with diminished denture stability and retention, difficulties tolerating prostheses, limited bite force, and compromised facial esthetics. 6

\section{Dental Caries}

Caries are classified depending on which surfaces of the tooth have been affected (Figure 2). Coronal caries occurs when the enamel and dentin of the coronal portion (the crown) of the tooth are affected. Dentate older adults are susceptible to coronal caries caused both by recurrent decay

\section{Table 1. Oral Tissues and Their Functions}

Oral Tissue

Teeth

Periodontium

Salivary glands

Taste buds

Oral mucosa

Muscles of mastication and facial expression

\section{Function}

Mastication, bone regeneration

Mastication, bone regeneration, host defense

Lubrication, oral mucosal repair, antimicrobial activity, remineralization of teeth, mediation of taste

Taste, host defense

Host defense, mastication, swallowing, speech

Mastication, swallowing, speech, posture 


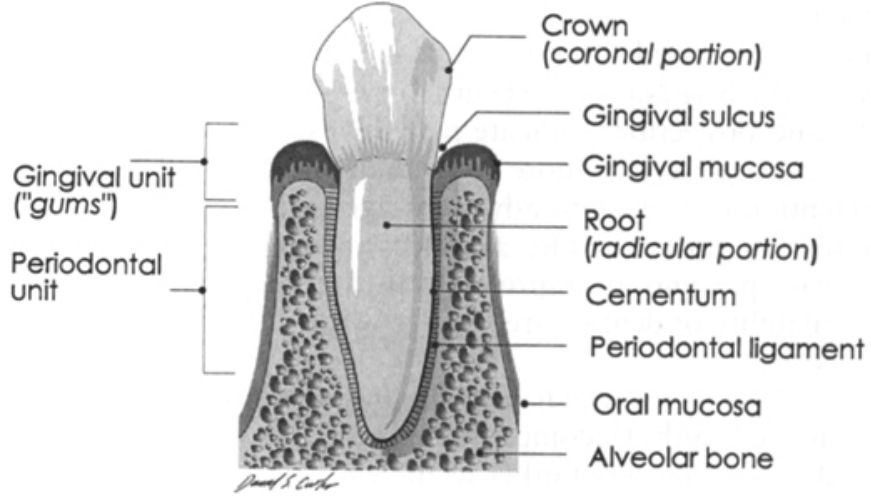

Figure 2. Schematic of dental and periodontal anatomic terms used in the text.

around existing restorations (fillings, crowns, etc.), and by new-onset decay of the coronal portion of the teeth. ${ }^{25}$ Root surface or "cervical" caries may occur when active or prior periodontal disease (see below) has caused the root surfaces of the tooth to become exposed to the oral environment. Root surface caries increases dramatically with age because of greater prevalence and severity of such periodontal conditions in older persons. ${ }^{15,26}$

The principal (though by no means the only) microorganism causing coronal caries in humans is Streptococcus mutans. ${ }^{27}$ Oral streptococci, actinomycetes, and lactobacilli are organisms commonly associated with cervical lesions. ${ }^{28}$ Caries-causing bacteria reside on the tooth surface in an adherent microbial colony (dental plaque). In the presence of sucrose and other simple sugars, bacteria of the plaque produce lactic acid, resulting initially in demineralization of tooth mineral and eventually in irreversible destruction of tooth structure. ${ }^{27}$ Individuals of all ages are susceptible to the development of dental plaque. However, following abstention from daily oral care, older individuals form plaque more rapidly than do younger people, ${ }^{29}$ possibly because they are more likely to have gingival recession, diminished salivary gland function (see below), disturbances in oral motor function, and difficulty performing oral hygiene. ${ }^{3}$ Since these factors are directly associated with dental caries, older patients are more likely to develop new and recurrent tooth decay.

Adult tooth surfaces can become resistant to decalcification and decay through repeated exposure to fluoride in water supplies, toothpaste, rinses, and gels. Yet even resistant tooth surfaces can become carious when oral hygiene is inadequate and there are dietary sources of fermentable carbohydrate. ${ }^{27}$ When detected early, caries can be debrided from a tooth and the missing tooth structure restored with a wear-resistant, insoluble dental material (e.g., silver amalgam, composite resin). ${ }^{30}$ Untreated dental caries, however, will in most circumstances progress to severe or even total loss of tooth structure, and possibly pain, abscess formation, cellulitis, and bacteremia.

\section{Periodontal Diseases}

The periodontium consists of those tissues that invest and support the tooth (see Figure 2). Dental plaque is considered to be the principal source of pathogenic organisms in periodontal diseases. ${ }^{31}$ Gingivitis occurs when bacterial byproducts and irritation cause inflammation of the gingival unit. Under certain host-mediated conditions, the microbial species or their byproducts cross the gingival epithelium and enter subepithelial tissues, where specific host-defense mechanisms and osteoclastic activity are initiated. This state, periodontitis, eventually results in tissue destruction, including bone loss and tooth mobility. The loss of the connective tissue attachment between cementum and bone is termed attachment loss. In the dominant form of periodontitis, termed "chronic adult periodontitis," repeated, brief periods of periodontal destruction throughout adulthood are interspersed with intervals absent from inflammation, resulting in a net loss of attachment that increases with time.

Periodontal diseases and their most serious result, attachment loss, are among the most prevalent of all diseases known to humans. With increased age, some gingival recession and loss of periodontal attachment and bony support are essentially universal: nearly $95 \%$ of dentate Americans over the age of 65 years have measurable attachment loss. ${ }^{15}$ Yet changes in the periodontium attributed solely to age are not sufficient to lead to tooth loss. ${ }^{32,33}$ However, because multiple oral factors, systemic diseases, and medications have an adverse influence on periodontal health, and because these conditions are more prevalent among older adults, they are at a greater risk for experiencing morbidity as a result of periodontal diseases.

For example, studies implicate diabetes as a risk factor for developing periodontal disease, ${ }^{34}$ and adult subjects with diabetes mellitus have a higher prevalence and severity of periodontal disease than age-matched healthy controls. Several classes of medications frequently prescribed in older people have been associated with gingival overgrowth or gingival hyperplasia, a condition that left untreated predisposes to both caries and destructive periodontitis. ${ }^{35}$ The family of calcium channel blockers, including diltiazem, verapamil, nifedipine, felodipine, nitrendipine, and oxodipine, can cause this unwanted drug side effect, which may require resective periodontal surgery for definitive treatment. The antiseizure medication phenytoin and the immunosuppressant cyclosporin have also been associated with severe cases of this condition. ${ }^{36}$

Periodontal diseases have oral and systemic effects on health. They are directly associated with halitosis, gingival bleeding, tooth mobility, and tooth loss. These outcomes can adversely affect food choice, mastication, swallowing, interpersonal relationships, and ultimately the nutritional intake of an individual. ${ }^{37}$ Untreated periodontitis, a chronic infection, may interfere with blood glucose control in diabetic patients. ${ }^{38}$ Recent epidemiologic studies report significant correlation between cardiovascular disease and periodontitis after controlling for such traditional risk factors as weight, gender, tobacco use, age, and blood lipid levels. ${ }^{39}$ Gramnegative bacteria are implicated in the pathogenesis of periodontal disease, ${ }^{31}$ and colonization of the oropharynx with Gram-negative bacilli predisposes to Gram-negative bacillary pneumonia. ${ }^{14}$ These and other periodontal pathogens have also been associated with bacteremia, ${ }^{40}$ infective endocarditis, and brain abscess. ${ }^{41}$

\section{Oral Mucosal Diseases}

In health, the mucosal tissues of the oral cavity contain the food bolus, control the flow of air in speech production, and serve as an important barrier against microbial invasion of the host. The mucosal tissues also provide the brain with 
the dominant share of afferent tactile and thermal input from the mouth. For these reasons, diseases of the oral mucosa can be seriously debilitating and disruptive.

The oral mucosa is a common site for vesiculobullous, desquamative, ulcerative, and lichenoid lesions. Pemphigus vulgaris, cicatricial and bullous pemphigoid, lichen planus, recurrent aphthous stomatitis, Herpes simplex and Herpes zoster may present with painful oral and perioral lesions. ${ }^{8}$ Many medications prescribed for older patients can induce intraoral mucosal reactions. ${ }^{42}$ Cytotoxic antineoplastic agents commonly cause oral sloughing and subsequent painful bacterial and fungal colonization. Oral ulcerations are reported side effects of furosemide, hydrochlorothiazide, triamterene, and spironolactone, as well as of the $\beta$-blockers propranolol, metoprolol, atenolol, and timolol. Stomatitis has been reported by patients on cholestyramine, piroxicam, and desipramine. Prazosin and clonidine can both be responsible for reactions similar to oral lichen planus. The ACEinhibitors captopril, lisinopril, and enalapril have all been associated with glossitis, and doxepine with aphthous stomatitis.

Candida albicans and (less commonly) C. glabrata are commensal fungi of the oral cavity that may become focally or systemically pathogenic under certain conditions. These conditions may include use of inadequately cleaned and/or ill-fitting dentures, suppression of normal oral flora as a result of broad-spectrum antibiotic use, impaired salivary flow (resulting in lowered oral $\mathrm{pH}$ and absence of fungistatic salivary histatins), use of corticosteroid inhalers, and immunocompromising conditions (e.g., diabetes, leukemias, AIDS)..$^{43}$ Tingling, burning pain, and taste disturbance may be reported. Oropharyngeal candidiasis is highly prevalent in older people, particularly in denture-wearing and institutionalized older adults. Budzt-Jörgensen diagnosed the condition in more than $65 \%$ of denture-wearing community-dwelling older people, ${ }^{44}$ and rates as high as 68 to $88 \%$ have been reported in institutionalized older populations. ${ }^{45,46}$ Although the disease is often asymptomatic, it is a pathological condition, and its presence signals the need to rule out potentially serious underlying causes.

More than $95 \%$ of oral malignancies are squamous cell carcinoma. Three out of four cases of oral-pharyngeal cancer occur in individuals 50 years of age and over. ${ }^{9}$ More than 28,000 new cases of oral cancer and about 8400 deaths caused by it are estimated in the United States for 1995, accounting for approximately $3 \%$ of cancer diagnoses (and $2 \%$ of cancer deaths) in males and $2 \%$ of cancer diagnoses ( $1 \%$ of cancer deaths) in females. Oral cancer is more prevalent than leukemia or malignancy of the stomach, cervix, esophagus, pancreas, liver, thyroid, brain, and ovary. ${ }^{47}$ The major risk factors associated with oral cancer are advanced age, male gender, previous oral malignancy, use of tobacco and alcohol, and exposure to sunlight (for cancer of the lip). When diagnosed before lymph node involvement has occurred, management results in limited morbidity and impairment, and 5-year survival rates range from $50 \%$ (for tongue) to $95 \%$ (for lip). ${ }^{9}$ With nodal and metastatic spread, survival drops precipitously, and the morbidity associated with treatment (e.g., surgical resection, irradiation, and cytotoxic chemotherapy) becomes pronounced, often involving disfigurement, speech impediment, salivary gland hypofunction, and osteomyelitis. For high-risk older patients (e.g, smokers and heavy drinkers), early detection through annual oral examination is imperative. ${ }^{48}$

\section{Salivary Function and Dysfunction}

There are three major pairs of salivary glands (parotid, submandibular, sublingual) and several groups of minor glands (e.g., labial, palatal, buccal) whose principal function is the exocrine production of saliva. In health, saliva contains many constituents that are critical to the maintenance of oral health, including those involved in lubrication of the oral mucosa, in remineralization of teeth, and in protection against viral, bacterial, and fungal infections. ${ }^{10}$

Recent studies have revealed that in healthy older adults there is no general diminution in the volume of saliva produced. ${ }^{49}$ Furthermore, there appear to be no significant alterations in the composition of saliva in older persons. ${ }^{50} \mathrm{How}$ ever, many older persons complain of a dry mouth (xerostomia) and have decreased salivary output. These problems are most likely caused by systemic diseases and their treatments rather than by the normal sequelae of aging. ${ }^{51}$ For example, many medications taken by older persons reduce or alter salivary gland performance. These include anticholinergic, antihypertensive, anxiolytic, antidepressant, diuretic, and antihistaminic preparations. Additionally, common forms of oncological therapy, such as radiation for neoplasms of the head and neck and cytotoxic chemotherapy, can have deleterious effects on salivary glands. The single most common disease specifically affecting salivary glands is Sjögren's syndrome, an autoimmune exocrinopathy occurring mainly in postmenopausal women.

All of the major physiological roles for saliva in the oral cavity can be adversely affected by its hyposecretion, regardless of etiology. ${ }^{52}$ With salivary gland dysfunction, increased dental caries will ensue rapidly because of the loss of the antimicrobial, buffering, and diluting properties of saliva. The greater adherence of plaque in the absence of adequate saliva creates an increased risk of developing periodontal diseases. The oral mucosa becomes desiccated and cracked, leaving the host more susceptible to microbial infection. Salivary gland dysfunction can also lead to difficulty in swallowing or speaking, pain (which may arise from either the teeth or the oral soft tissues), and diminished enjoyment of food. Finally, there is evidence that the lack of saliva may increase the risk of aspiration pneumonia (see below) and cause esophageal and gastrointestinal problems. ${ }^{53,54}$

\section{Oral Motor Function}

The oral motor apparatus is involved in several routine yet intricate functions (see Table 1). Regulation of these activities may occur at three levels: the local neuromuscular unit, central neuronal pathways, and systemic influences. Furthermore, studies of oral motor function have shown that some alterations in performance (mastication, swallowing, oral muscular posture, and tone) can be expected with increasing age. ${ }^{11,24,55,56}$ The most often reported oral motor disturbance in older people is related to altered mastication, and even fully dentate older persons are less able to prepare food for swallowing as efficiently as younger individuals. ${ }^{19,24}$ This altered masticatory ability in older age can be exacerbated further among individuals with partial or total edentulousness, teeth rendered mobile or painful by periodontal diseases, and decreased salivary output. 
Following mastication, the food bolus is translocated to the pharynx. This oral phase of swallowing requires well coordinated neuromuscular processing, an intact mucosal barrier, and adequate saliva production. Alterations in any of these components can disturb deglutition ${ }^{57,58}$ and reduce nutritional intake. ${ }^{55}$ Although the oral phase of swallowing undergoes only subtle changes with increased age, under unusual or stressful conditions these perturbations can place older persons (especially those with dentures) at some risk of choking or aspiration.

Oral motor function also contributes to the production of speech, which undergoes changes with age. Specifically, there are alterations in tongue shape and function during production of specific speech sounds ${ }^{57}$ and variability in frequency of speech patterns. ${ }^{59}$ However, among healthy older persons, these changes do not compromise or alter speech in any perceptible way. ${ }^{57}$ There are also ageassociated alterations in intraoral and maxillofacial posture. Many older persons suffer from drooping of the lower face and lips and experience some difficulty with closing the lips competently while eating, drinking, or swallowing. Drooling in an older person may also be caused by reduced circumoral muscle tone and/or delayed swallowing. More commonly, exacerbations of these oral motor states are caused by systemic diseases (e.g., cerebrovascular accidents and Parkinson's disease) and therapeutic drug regimens (e.g., the association of tardive dyskinesia with phenothiazine therapy). ${ }^{60}$

\section{Sensory Function}

Many older adults complain of diminished food recognition and enjoyment, as well as altered smell and taste function. Perturbations in taste and smell or other oral sensory modalities (temperature, viscosity, pressure) may occur with increased age ${ }^{61}$ and reduce the rewards of eating, thus contributing to a diminished interest in food.

Recent studies have objectively evaluated gustatory function in generally healthy persons of different ages, and have found no overall decremental changes. ${ }^{62}$ Conversely, objective studies of olfaction reveal dramatic age-related changes. ${ }^{63}$ Other investigations have evaluated the more complicated problems of flavor perception, food recognition, and food preference. Although results are not uniform, older individuals do less well when performance is assessed in these tasks. ${ }^{62}$ It appears that chemosensory functions of older individuals are compromised by diminished olfactory performance, which can lead to a diminished interest in food. Furthermore, because sensory function plays an important role in warning the host of fire, natural gas, toxic vapors, and spoiled foodstuffs, older people may be at increased risk for failing to detect these potentially hazardous materials.

While older age has been associated with oral sensory problems, many oral and systemic conditions have been linked more strongly to chemosensory dysfunction. ${ }^{12}$ Dental/ alveolar abscesses, salivary gland dysfunction, periodontal diseases, oral candidiasis, and the utilization of removable dental prostheses can cause taste and smell problems. In addition, multiple systemic diseases (e.g., Alzheimer's disease and Parkinson's disease), medications (e.g., captopril and ferrous sulfate), and oncological therapies (e.g., head and neck irradiation and cytotoxic chemotherapy) can cause chemosensory disorders. ${ }^{64,65}$

There are significant oral and systemic sequelae of taste and smell problems, including nutritional deficits, ${ }^{66}$ and in- dividuals with these problems suffer from a diminished quality of life. Because oral diseases in older people have been associated with nutritional deficiencies, ${ }^{22}$ it is likely that chemosensory dysfunction will increase the risk of an older person developing a significant nutritional disorder.

\section{SYSTEMIC EFFECTS OF ORAL BACTERIA}

The oral cavity supports a diverse microbiotia. Microenvironmental niches such as the buccal mucosa, tongue dorsum, gingival sulcus, and the teeth each support their own microbiological communities in health ${ }^{41,67}$ (Table 2). In the presence of oral disease and in association with fixed and removable prosthetic devices, additional microenvironments may be present. The composition of the bacterial colonies of the teeth and gingival sulcus in particular are dynamic and reflect the combined influences of host defense, oral hygiene, and the presence or absence of dental and periodontal diseases. ${ }^{41}$ The pathogenic impact of oral bacteria is generally restricted to the mouth and perioral structures by hostprotective mechanisms such as epithelial barriers, specific and nonspecific antimicrobial properties of saliva (e.g., immunological and nonimmunological aggregating factors, lysozyme, lactoferrin, and salivary peroxidase), ${ }^{10}$ and the surveillance of the immune system. But dental and periodontal bacteria spread by bacteremia or aspiration can be the source of life threatening diseases. For this reason, the aforementioned trend for increased tooth retention in advanced age should be viewed as a sign of improved health that nevertheless carries its own set of risks.

Bacteremia of oral origin is readily induced by manipulation of teeth or gingiva, such as tooth extraction, periodontal scaling, flossing, and tooth brushing. ${ }^{40,68,69}$ Even chewing can result in cultivable blood-borne oral bacteria in the host with gingival and/or periodontal inflammation. ${ }^{40}$ Bacteremia of oral origin may be of little acute significance in the healthy host. But for many older adults, the use of implanted devices, cardiac flow abnormalities, and/or increased susceptibility to infection make the potential consequences of bacteremia particularly dire. ${ }^{70}$ The American Heart Association has published recommendations for antibiotic prophylaxis before many dental procedures performed on patients with certain cardiac conditions and valve replacements. ${ }^{71}$ The need for similar preventive approaches to patients with other conditions is less well supported. For instance, more than 119,000 total hip arthroplasties are placed annually in the United States, ${ }^{72}$ and orthopedists strongly favor the use of prophylactic antibiotics before dental treatment for patients with these implanted devices. ${ }^{73}$ Nevertheless, clinical and experimental data have not established whether such measures are indicated, or, if so, what agent and dosage schedule is optimal. ${ }^{74}$ There is even less information regarding the management of patients with venous catheters, ventricular shunts, or organ transplants.

Aspiration of oral secretions and their bacteria is increasingly being recognized as an important factor in pneumonia. Normative decrements in swallowing function ${ }^{11}$ and ageprevalent diseases that cause dysphagia (e.g., stroke, Parkinson's disease, and Alzheimer's disease) predispose to repeated aspiration of oral contents. As stated previously, bacteria implicated in the pathogenesis of periodontal disease can colonize the oropharynx with Gram-negative bacilli. Further, salivary gland hypofunction places an individual at increased 
Table 2. Common Oral Bacteria, Associated Oral and Non-oral Pathoses, and Oral Distribution ${ }^{41,67}$

Relative Distribution in

Different Sites of the

Organism

Oral Pathosis

Non-oral Pathosis Oral Cavity*

Faculative, Gram (+) cocci

Streptococcus salivarius

S. mitis

S. sanguis

S. mutans

S. faecalis (Enterococcus)

S. milleri

S. pyogenes

Anaerobic, Gram (+) cocci

Peptostreptococcus species

Facultative, Gram (-) cocci

Neisseria species

Anaerobic Gram (-) cocci

Veillonella species

Facultative Gram (+) rods

Lactobacillus species

Anaerobic, Gram (-) rods

Bacteroides species

Vibrio sputorum

Fusobacterium

Facultative, Gram (-) rods

Haemophilus

parainfluenza

Actinobacillus

actinomycetemcomitans

Filaments, Gram (+)

Actinomycetes

Clostridium species

Spirochetes: Treponema

\begin{abstract}
coronal caries
dental abscess

dental abscess

dental abscess
\end{abstract}

pulpitis

root caries

periodontitis, gingivitis

ulcerative gingivitis

juvenile periodontitis

root caries

orofacial infection following

trauma

acute necrotizing ulcerative

gingivitis, periodontitis infective endocarditis

infective endocarditis

pharyngitis, rheumatic

fever, glomerulonephritis

lung abscess, brain abscess

pleuropulmonary infection (rare)

pneumonia, infective endocarditis

bacteremia and sequelae; upper respiratory infection

upper respiratory infection

$T=S>G$

G

$P>G>T>S$ cervicofacial and thoracic actinomycosis;

bacteremia and

sequelae; brain abscess

$$
\begin{aligned}
& T=S>B \\
& B>S>P>T=G \\
& P>B>S=G>T \\
& P>S \\
& G=T=S \\
& G>S \\
& P \\
& P=S>G>T \\
& T=B=S \\
& T>G=S>P=B \\
& P>S
\end{aligned}
$$

$G>P=T$

- $\mathrm{G}=$ gingival sulcus; $\mathrm{P}=$ coronal plaque; $\mathrm{T}=$ tongue dorsum; $\mathrm{B}=$ buccal mucosa; $\mathrm{S}=$ saliva

risk for oropharyngeal Gram-negative colonization, ${ }^{75}$ which can result in bacillary pneumonia. ${ }^{14}$

\section{THE NON-DENTIST'S CONTRIBUTION TO THE OLDER PATIENT'S ORAL HEALTH}

Oral health is inextricably linked to the overall health of the older patient because stomatologic diseases have consequences that extend beyond the confines of the mouth. Moreover, many stomatologic conditions in older people are caused or worsened by systemic diseases and their treatments rather than by the aging process alone. Therefore, the health care provider who diagnoses and treats older patients' medical conditions should strive to identify individuals at risk for developing oral problems and collaborate with oral health specialists to treat and prevent oral disease. Patients of all ages report more physician visits per year than dental visits, and this difference increases with increasing age ${ }^{76,77}$ (Figure 3). Inasmuch as most oral diseases are preventable and treatable, physicians and nurses caring for older patients need either to recruit dental expertise routinely as part of their overall assessment or to familiarize themselves with the appearance of oral health and diseases states. ${ }^{78}$ Dentists should refer patients to physicians for previously undiscovered or inadequately controlled medical problems such as diabetes, hypertension, and cardiovascular disease, and often need to draw on medical expertise in order to provide dental treatment at minimal risk to the patient. Similarly, patient wellbeing is also optimized when non-dentists detect oral disease 


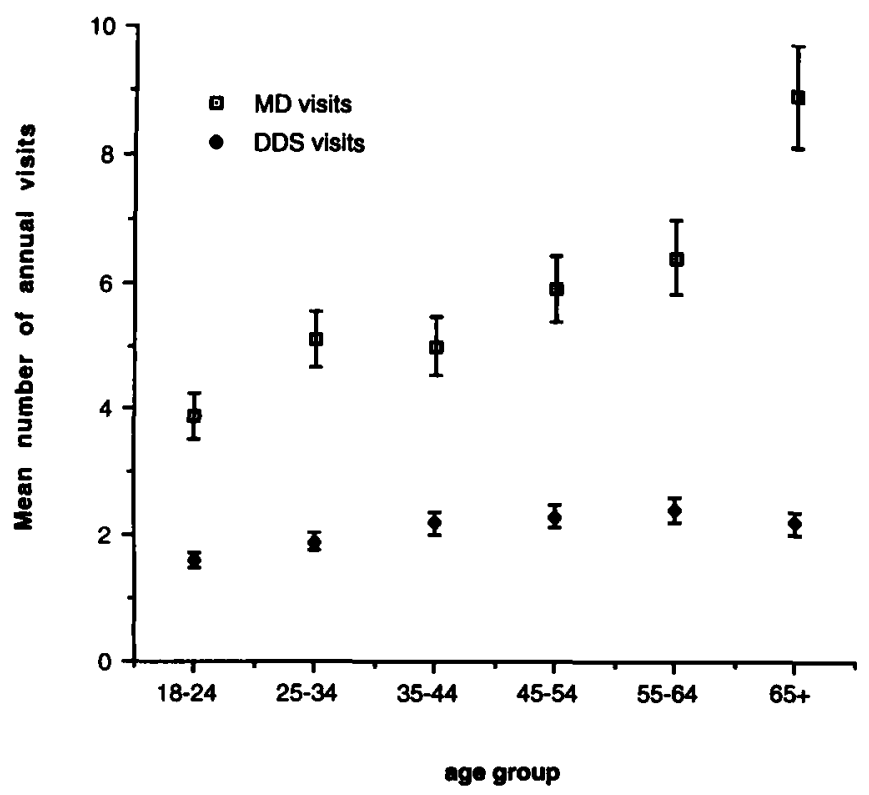

Figure 3. Relationship between age group and mean number of annual patient visits to physician and to dentists, based on the 1989 National Health Interview Survey. ${ }^{76,77}$ Error bars reflect maximum calculated estimates of standard errors of the mean for each age group. Analysis of variance demonstrates statistically significant increase $(P=.0007)$ in the difference between number of physician visits and dentist visits as a function of age.

and recommend attention to preventive and interventive treatment.

Clinicians need to take into account the significance of oral side effects of drugs prescribed for non-oral conditions. Painful mucositis can impair nutritional intake and undermine the patient's ability to complete a course of chemotherapy. Medications that interfere with oral health by contributing to salivary hypofunction or gingival hyperplasia signal a need to consider a different therapeutic regimen. In cases where an alternative is not feasible, prescription of such agents should be accompanied by a referral to a dentist to mitigate the oral impact of the drug.

Although it is important that geriatric health providers recognize the relationships between oral and systemic health, that knowledge alone will not be sufficient to improve oral health. Providers must share this information with patients, stressing the fact that oral diseases are not unavoidable concomitants of aging and that they can be prevented. As other aspects of healthy lifestyle and preventive health are advocated for patients, so should be limitation of refined sugar intake, performance of daily oral hygiene (including use of a fluoride dentifrice), and regular dental visits.

Finally, there are, at present, serious financial constraints to obtaining dental care. ${ }^{79}$ The number of annual dental visits is strongly positively correlated with income, although the number of annual physician visits is independent of that factor $^{76,77}$ (Figure 4). Medical insurance is a strong predictor for use of dental services (Figure 5), yet fewer than $15 \%$ of people older than age 65 have private dental insurance, compared with about $45 \%$ for Americans of all ages. Medical insurance seldom, if ever, includes coverage for dental procedures. Medicare currently covers essentially no dental costs. Medicaid reimbursement for adult dentistry is available in fewer than half of the states and, where offered, covers at

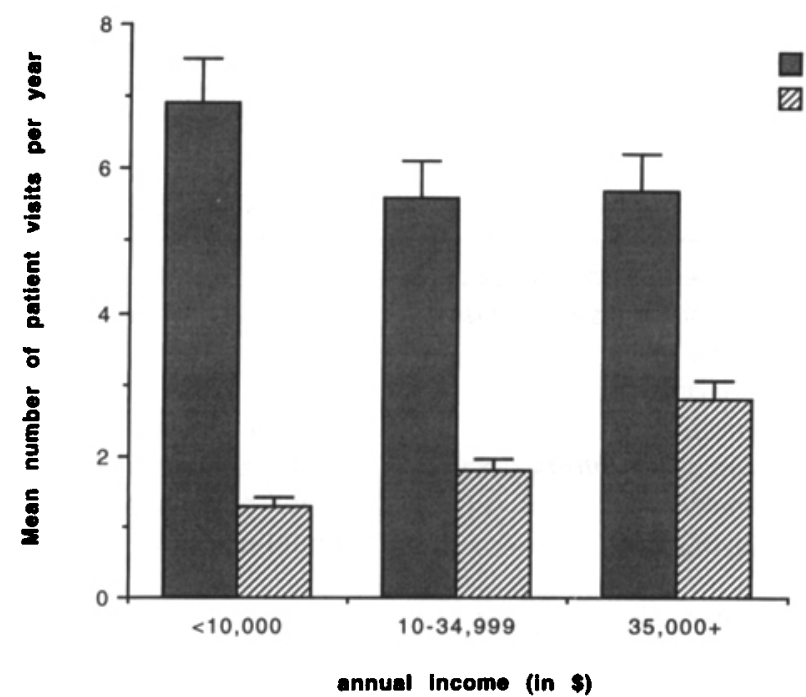

Figure 4. Relationship between annual patient income and mean number of annual patient visits to physicians and to dentists, based on the 1989 National Health Interview Survey. ${ }^{76,77}$ Error bars reflect maximum calculated estimates of standard errors of the mean for each age group. Analysis of variance demonstrates no statistically significant income effect for physician visits, but a strong positive relationship between the number of dentist visits and patient income $(P<.005)$.

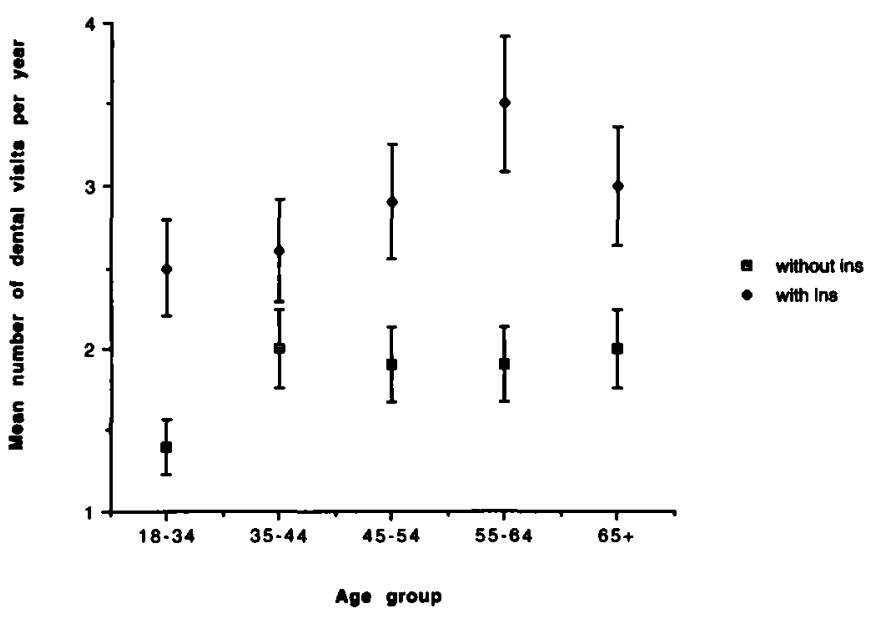

Figure 5. Relationship between private dental insurance status ("ins"), age group and mean number of annual patient visits to dentists, based on the 1989 National Health Interview Survey. ${ }^{77}$ Error bars reflect maximum calculated estimates of standard errors of the mean for each age group.

state-specific rates that many dentists consider unacceptably low. In light of the central importance of oral health to an older patient's overall well-being, it is essential that all health providers support and advocate the expansion of oral health benefits for older adults.

\section{SUMMARY}

Oral health is important for general health because stomatologic diseases affect more than the mouth. Furthermore, systemic conditions can have a strong impact on oral health and function. Oral problems in advanced age are largely attributable to preventable diseases rather than the aging process per se. Stomatologic disease and dysfunction impact older persons' general health, making oral health a concern 
for all who care for them. Translating that concern into positive benefits for older patients requires effective interdisciplinary collaboration, patient education, and advocacy for dental care.

\section{ACKNOWLEDGMENTS}

The authors are grateful to Teresa Dolan, DDS, MPH (University of Florida, Gainesville) and Edmund H. Duthie, Jr., MD (Medical College of Wisconsin) for their thoughtful comments on this manuscript. Andrzej T. Galecki, MD, PhD of the University of Michigan Institute of Gerontology and Van Parsons, PhD of the National Center for Health Statistics provided invaluable guidance and assistance in the analysis of the National Health Interview Survey data.

\section{REFERENCES}

1. Gift HC, Redford M. Oral health and the quality of life. Geriatr Clin North Am 1992;8:673-683.

2. Beck JD. Epidemiology of dental diseases in the elderly. Gerodontology 1984;3:5-15.

3. Baum BJ, Ship JA. Oral disorders. In: Beck J, ed. Geriatrics Review Syllabus - A Core Curriculum in Geriatric Medicine. New York: American Geriatrics Society, 1991, pp 332-336.

4. Lloyd PM, Shay K. The how and why of tooth loss in the elderly. Part I: The uncompensated tooth loss syndrome. Geriatr Dent Update 1988;2:1-3.

5. Tallgren $A$. The continuing reduction of the residual alveolar ridges in complete denture wearers: A mixed-longitudinal study covering 25 years. J Prosthet Dent 1972;27:120-132.

6. Ettinger RL. Managing and treating the atrophic mandible. J Am Dent Assoc 1993;124:234-241.

7. Budzt-Jörgensen E. Clinical aspects of Candida infection in denture wearers. J Am Dent Assoc 1978;96:474-479.

8. Silverman S. Oral defenses and compromises: An overview. NCI Monographs 1990;9:17-19.

9. Silverman S. Oral Cancer, 3rd Ed. Atlanta: American Cancer Society, 1990.

10. Mandel ID. The role of saliva in maintaining oral homeostasis. J Am Dent Assce 1989;119:298-304.

11. Robbins J, Hamilton J, Lof $\mathrm{C}_{\text {, }}$ Kempster $\mathrm{G}$. Oropharyngeal swallowing in normal adults of different ages. Gastroenterology 1992;103:82.3-829.

12. Ship JA. Gustatory and olfactory considerations: Examination and treatment in general practice. J Am Dent Assoc 1993;124:55-62.

13. Mulligan $R$, Navazesh $M$. Relationship between oral conditions and sysremic diseases in the elderly. J Dent Res 1992;71 (Spec Iss) (Abstract) 1681:316.

14. Bartlett JG. Pneumonia. In: Hazzard WR, Bierman EL, Blass JP et al, eds. Principles of Geriatric Medicine and Gerontology, 3rd Ed. New York: McGraw-Hill, 1994, pp 565-573.

15. Miller AJ, Brunelle JA, Carlos JP et al. Oral health of United States adults. U.S. Department of Health and Human Services. NIH publication no. 872868. National Institutes of Health, Public Health Service. Washington DC: Government Printing Office, 1987.

16. Bailit HL, Braun R, Maryniuk GA, Camp P. Is periodontal disease the primary cause of tooth extraction in adults? J Am Dent Assoc 1987;114:4045.

17. Ship JA, Ship II. Trends in oral health in the aging population. Dent Clin North Am 1989;33:33-42.

18. Ettinger RL. Cohort differences among aging populations: A challenge for the dental profession. Spec Care Dent 1993;13:19-26.

19. Wayler AH, Muench ME, Kapur KK, Chauncey HH. Masticatory performance and food acceptability in persons with removable partial dentures, full dentures and intact natural dentition. J Gerontol 1984;39:284-289.

20. Heath MR. Dietary selection by elderly persons, related to dental state. $\mathrm{Br}$ Dent J 1972;132:145-148.

21. Chen MK, Lowenstein F. Masticatory handicap, socioeconomic status, and chronic conditions among adults. J Am Dent Assoc 1984;109:916-918.

22. Posner BM, Jette A, Smigelski C et al. Nutritional risk in New England elders. J Gerontol Med Sci 1994;49:M123-132.

23. Litvak $H$, Silverman $S$, Garfinkel L. Oral stereognosis in dentulous and edentulous subjects. J Prosthet Dent 1971;25:139-151.

24. Feldman RS, Kapur KK, Alman TE, Chauncey HH. Aging and mastication: Changes in performance and in the swallowing threshold with natural dentition. J Am Geriatr Soc 1980;28:97-103.
25. Beck JD, Hunt RJ, Hand JS Field HM. Prevalence of root and coronal caries in a noninstitutionalized older population. J Am Dent Assoc 1985;111:964967.

26. Galan D, Lynch E. The epidemiology of root caries. Gerodontology 1993;10:59-71.

27. Shaw JH. Causes and control of dental caries. N Engl J Med 1987;317:9961004.

28. Bowden GHW. Microbiology of root surface caries in humans. J Dent Res 1990;69:1205-1210.

29. Holm-Pedersen P, Agerback N, Theilade E. Experimental gingivitis in young and elderly individuals. J Clin Periodontol 1975;2:14-24.

30. Berkey DB, Shay K. General dental care for the elderly. Geriatr Clin North Am 1992;8:579-597.

31. Genco RJ. Using antimicrobial agents to manage periodontal diseases. J Am Dent Assoc 1991;122:31-38.

32. Ainamo A, Ainamo J. The dentition is intended to last a lifetime. Int Dent J 1984;34:87-92.

33. Burt BA. Periodontitis and aging: Reviewing recent evidence. J Am Dent Assoc 1994;125:273-279.

34. Schlossman M, Knowler WC, Pettitt DM et al. Type 2 diabetes mellitus and periodontal disease. J Am Dent Assoc 1990;121:532-536.

35. Brown RS, Beaver WT, Bottomley WK. On the mechanism of drug-induced gingival hyperplasia. J Oral Pathol Med 1991;20:201-209.

36. Hassell TM, Hefti AF. Drug-induced gingival overgrowth: Old problem, new problem. Crit Rev Oral Biol Med 1991;2:103-137.

37. Anderson EL. Eating patterns before and after dentures. J Am Dietetic Assoc 1971;58:421-426.

38. Samalkorpi K. Glucose intolerance in acute infections. J Intern Med 1989;25:15-19.

39. DeStefano F, Anda RF, Kahn HS et al. Dental disease and risk of coronary heart disease and mortality. Br Med J 1993;306:688 -691.

40. Sconyers JR, Crawford JJ, Moriarty JD. Relationship of bacteremia to toothbrushing in patients with periodontitis. J Am Dent Assoc 1973;87:616-622.

41. Rosen S, Elvin-Lewis E. Oral microflora. In: Willett NP, White RR, Rosen $S$, eds. Essential Dental Microbiology. Norwalk: Appleton \& Lange, 1991, pp 318-340.

42. Felder RS, Millar SB, Henry RG. Oral manifestations of drug therapy. Spec Care Dent 1988;8:119-124.

43. Truhlar MR, Shay K, Sohnle P. Use of a new assay technique for quantification of antifungal activity of nystatin-incorporated in denture liners. J Prosthet Dent 1994;71:517-524.

44. Budzat-Jörgensen E, Stenderup A, Grabowski M. An epidemiologic study of yeasts in elderly denture wearers. Community Dent Oral Epidemiol 1975;3:115-119.

45. Cardash HS, Helft M, Shani A, Marshak B. The prevalence of Candida albicans in denture wearers in an Israeli geriatric hospital. Gerodontology 1989:8:101-107.

46. Wilkieson C, Samaranayake LP, MacFarlane TW et al. Oral candidosis in the elderly in long term hospital care. J Oral Pathol Med 1991;20:13-16.

47. Wingo PA, Tong T, Bolden S. Cancer statistics, 1995. Cancer 1995;45:8-30.

48. Fedele DJ, Jones JA, Niessen LC. Oral cancer screening in the elderly. J Am Geriatr Soc 1991;39:920-925.

49. Ship JA, Baum BJ. Is reduced salivary flow normal in old people? Lancer 1990;336:1507.

50. Wu AJ, Atkinson JC, Fox PC et al. Cross-sectional and longitudinal analyses of stimulated parotid salivary constituents in healthy, different-aged subjects. J Gerontol Med Sci 1993;48:M219-224.

51. Atkinson JC, Fox PC. Salivary gland dysfunction. Clin Geriatric Med 1992;8:499-511.

52. Fox PC, van der Ven P, Sonies BC et al. Xerostomia: Evaluation of a symptom of increasing significance. J Am Dent Assoc 1985;110:519-525.

53. Valdez IH, Fox PC. Part I: The role of saliva in digestion. Interactions of the salivary and gastrointestinal systems. Dig Dis 1991;9:12.5-132.

54. Valdez IH, Fox PC. Part II: Effects of salivary gland dysfunction on the gastrointestinal tract. Interactions of the salivary and gastrointestinal systems. Dig Dis 1991;9:210-218.

55. Sonies BC. Oropharyngeal dysphagia in the elderly. Clin Geriatr Med 1992;8:569-577.

56. Baum BJ, Bodner L. Aging and oral motor function: Evidence for altered performance among older persons. J Dent Res 1983;62:2-6.

57. Sonies BC, Stone M, Shawker T. Speech and swallowing in the elderly. Gerodontology 1984;3:115-123.

58. Hughes CV, Baum BJ, Fox PC et al. Oral-pharyngeal dysphagia: A common sequelae of salivary gland dysfunction. Dysphagia 1987;1:173-176.

59. Benjamin BJ. Frequency variability in the aged voice. J Gerontol $1981 ; 36: 722-726$. 
60. Ship JA. Oral sequelae of common geriatric diseases, disorders, and impairments. Clin Geriatr Med 1992;8:483-497.

61. Weiffenbach JM, Tylenda CA, Baum BJ. Oral sensory changes in aging. J Gerontol Med Sci 1990;45:M121-125.

62. Weiffenbach JM, Bartoshuk LM. Taste and smell. Clin Geriatric Med 1992;8:543-555.

63. Doty RL, Shaman P, Applebaum SL et al. Smell identification ability changes with age. Science 1984;226:1441-1443.

64. Schiffman SS. Taste and smell in disease. N Engl J Med 1983;308:1275-1279, 1337-1343.

65. Schiffman SS. Drugs influencing taste and smell perception. In: Getchell TV, Bartoshuk LM, Doty RL et al., eds. Smell and taste in heaith and disease. New York: Raven Press, 1991, pp 845-50.

66. Murphy C. Nutrition and chemosensory perception in the elderly. Crit Rev Food Sci Nutr 1993;33:3-15.

67. Schonfeld SE. Oral microbial ecology. In: Slots J, Taubman MA, eds. Contemporary Oral Microbiology and Immunology. St Louis: Mosby Year Book, 1992, pp 267-282.

68. Crawford JJ, Sconyers JR, Moriarty JD et al. Bacteremia after tooth extraction studied with the aid of prereduced anaerobically sterilized culture media. Appl Microbiol 1974;27:927-932.

69. Baltch AL, Pressman HL, Schaffer C et al. Bacteremia in patients undergoing oral procedures. Arch Intern Med 1988;148:1084-1088.

70. Wahl MJ. Myths of dental-induced endocarditis. Arch Intern Med 1994;154:137-144.
71. Dajani AS, Bisno AL, Chung KJ et al. Prevention of bacterial endocarditis. Recommendations by the American Heart Association. JAMA 1990;264:2919-2922.

72. National Center for Health Statistics. Detailed diagnoses and procedures, national Hospital Discharge Survey, 1990. Vital and Health Statistics 1992, Series 13, no. 113.

73. Jaspers MT, Little JW. Prophylactic antibiotic coverage in patients with total arthroplasty: Current practice. J Am Dent Assoc 1985;111:943-948.

74. Jacobson JJ, Schweitzer SO, Kowalski CJ. Chemoprophylaxis of prosthetic joint patients during dental treatment: $A$ decision-utility analysis. Oral Surg Oral Med Oral Pathol 1991;72:167-177.

75. Gibson G, Barrett $E$. The role of salivary function on oropharyngeal colonization. Spec Care Dent 1992;12:153-156.

76. Ries $P$. Educational Differences in Health Status and Health Care. Vital and Health Statistics, Series 10, no. 179. DHHS Publication; no. (PHS) 91-1507, September 1991.

77. Bloom B, Gift HC, Jack SS. Dental services and oral health: United States, 1989. Vital and Health Statistics, Series 10, no. 183. DHHS Publication; no. (PHS) 93-1511, December 1992

78. Gordon SR, Jahnigen DW. Oral assessment of the dentulous elderly patient. J Amr Geriatr Soc 1986;34:276-281.

79. Meskin LH, Mason LD. Problems in oral health care financing for the elderly. Dent Clin North Am 1992;8:685-692.

80. National Center for Health Statistics: Edentulous persons, United States 1971. Data from the National Health Survey. Vital and Health Statistics 1974 series 10, no. 89. DHHS pub. no. (HRA) 74-1516. 